\title{
Caecal perforation in a renal transplant patient with disseminated histoplasmosis
}

\author{
MARY T BRETT, ${ }^{*}$ J T C KWAN, $†$ M R BENDING $\dagger$ \\ From the ${ }^{*}$ Department of Histopathology and the †South West Thames Regional Renal Unit, St Helier \\ Hospital, Carshalton, Surrey
}

SUMmARY A renal transplant patient developed a fatal caecal perforation after Histoplasma capsulatum infection acquired abroad. Disseminated histoplasmosis is an uncommon fungal infection, usually seen in patients with impaired immunity. The diagnosis should be considered in immunosuppressed patients who develop prolonged fever or whose health deteriorates unexpectedly after travelling overseas. The infection is endemic in parts of the United States of America but occurs all over the world. Rapid diagnosis is often possible by histological examination of infected tissues. Treatment if started early may lead to recovery, but if it is not treated it is usually fatal.

Fungal infection is a well known complication of immunosuppressive treatment. The causative organism is often Candida or Aspergillus, and occasionally a phycomycete such as Mucor. Overseas travel may expose immunosuppressed patients to other organisms that subsequently cause diseases infrequently seen in their countries of origin; the diagnosis may therefore be missed.

Histoplasmosis is endemic in parts of the United States but it occurs in many other countries.' Infection may disseminate, often in those with impaired immunity. ${ }^{23}$ We report a case of fatal caecal perforation in a renal transplant patient, and review published reports on the pathological features of disseminated histoplasmosis.

\section{Case report}

A 62 year old Asian man from Kenya had lived in England since 1963 and had developed end stage renal failure caused by chronic glomerulonephritis. He had a successful renal transplant in 1980 , and was taking prednisolone and azathioprine for immunosuppression. The graft was functioning well.

During a trip to Kenya in 1987 he developed abdominal pain, nausea, and vomiting. After returning home he had persistent dysphagia and nasopharyngeal pain, but barium swallow examination and indirect laryngoscopy showed no lesions. He also had intermittent abdominal pain and anorexia and lost weight.
Two months later he developed fever, cough, and pain in the right iliac fossa associated with profuse, foul smelling, watery diarrhoea. Physical examination showed abdominal distension and ascites. No organisms or cells were found in a specimen of ascitic fluid.

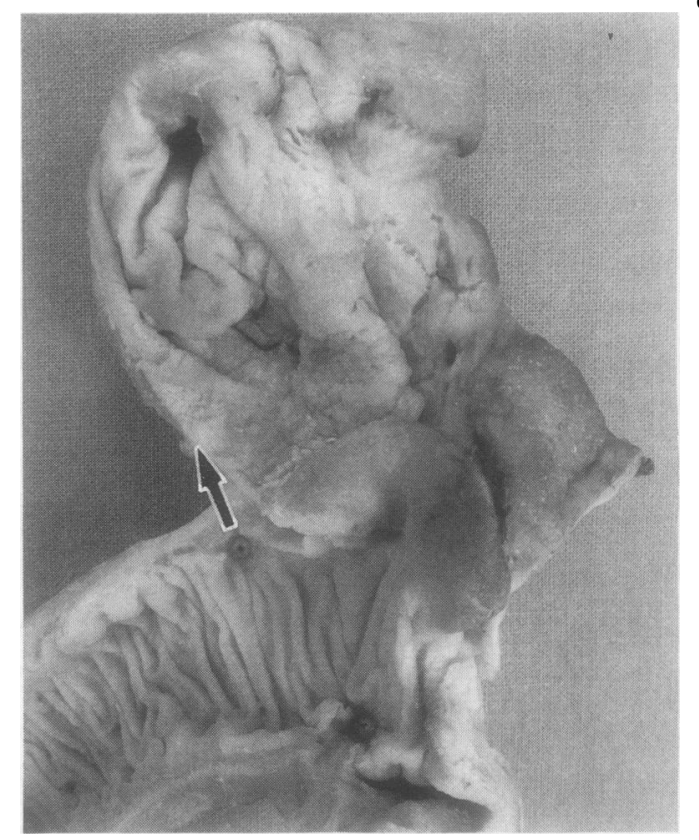

Fig 1 Part of caecum with polypoid lesion. Arrow points to edge of perforation. Terminal ileum is normal. 


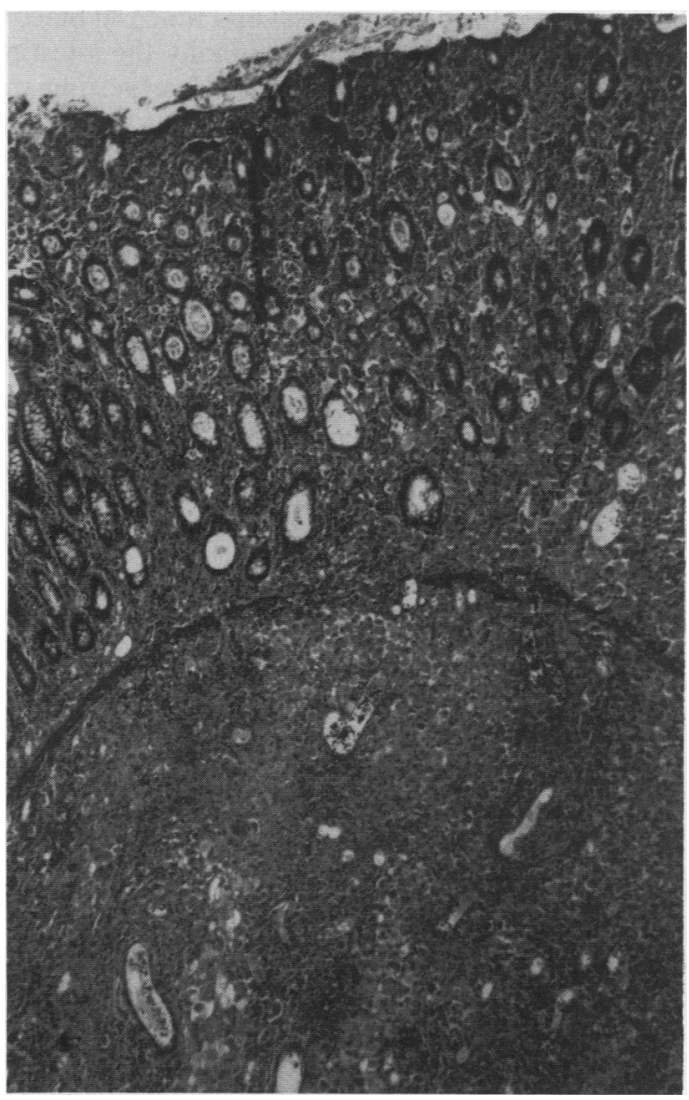

Fig 2 Polypoid lesion in caecum showing mucosal and submucosal expansion by sheets of macrophages.

(Haematoxylin and eosin.)

Sigmoidoscopy was normal, as was histologica examination of a rectal biopsy specimen, and no ova, cysts, or parasites were found in samples of stools. A chest radiograph showed a raised right hemidiaphragm and linear scars in the base of the left lung, both of which had been noted previously. His haemoglobin concentration was $10 \mathrm{~g} / \mathrm{dl}$, white cell count $2.8 \times 10^{9} / 1(82 \%$ neutrophils, $10 \%$ lymphocytes), and creatinine concentration $70 \mu \mathrm{mol} / \mathrm{l}$. Liver function tests were normal.

He rapidly developed peritonitis and paralytic ileus. At laparotomy a perforation in the posterior wall of the caecum was found, with pus in the pelvis; a right hemicolectomy was carried out. The remaining intestine and stomach looked normal. Despite inotropic support the patient died a few hours later. Permission for necropsy was not requested because he was seropositive for hepatitis $B$.

Subsequently, tests for Histoplasma antibodies in a serum sample taken before death showed a low titre of

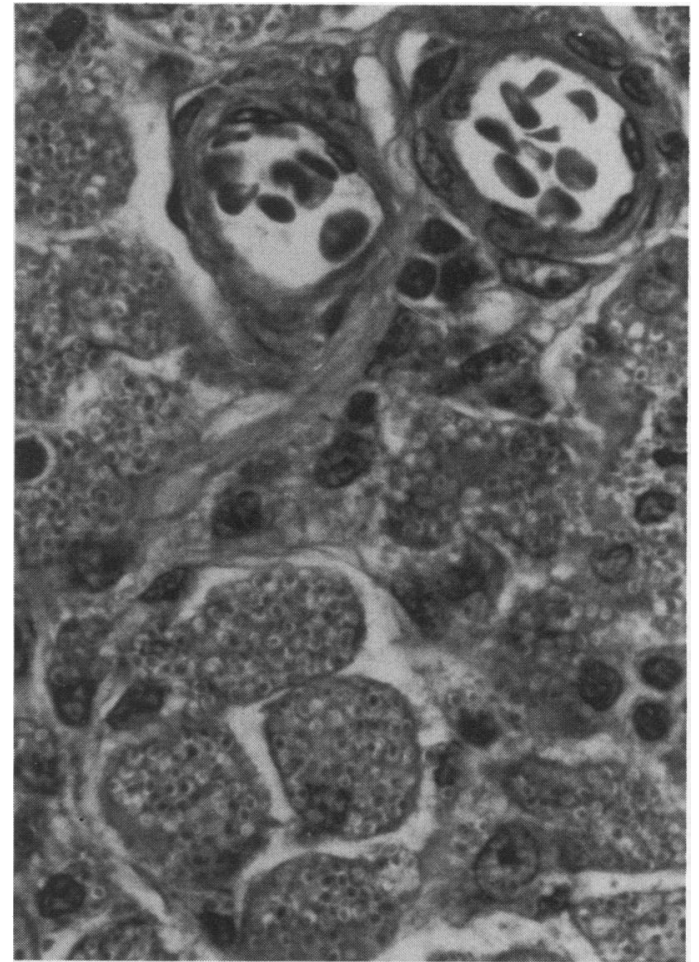

Fig 3 Macrophages in submucosa are filled with many $H$ capsulatum yeast forms, each with a clear halo. Compare with size of erythrocytes. (Haematoxylin and eosin.)

1:5 for yeast antibodies by complement fixation. These results were not confirmed by immunodiffusion or complement fixation for mycelial antibodies.

\section{HISTOPATHOLOGICAL FINDINGS}

The right hemicolectomy specimen showed a perforated caecal ulcer $80 \times 40 \mathrm{~mm}$ with polypoid edges (fig 1). The ascending colon contained several other polypoid lesions up to $20 \mathrm{~mm}$ in diameter, some showing superficial ulceration. The terminal ileum and appendix seemed normal.

Sections of formalin fixed, paraffin wax embedded tissue were stained with haematoxylin and eosin, periodic acid Schiff (PAS), methenamine silver (Grocott), alcian blue, and Giemsa stains. Histological examination of the ulcer and the polypoid lesions showed pronounced submucosal and mucosal expansion by macrophages (fig 2 ). They were packed with round, eosinophilic organisms $2-4 \mu \mathrm{m}$ in diameter, each with a clear halo (fig 3). The appearance in the haematoxylin and eosin, PAS, and Grocott stained sections was consistent with Histoplasma capsulatum. The organisms did not stain with alcian blue or Giemsa.

There was only a scanty lymphocytic infiltrate, and 
no granulomas were identified; there was necrosis in the areas of ulceration. There were yeast laden macrophages in the lymphatics, the pericaecal lymph node sinuses, and the serosal adipose tissue. The nodes were small without germinal centres, which probably reflected the effect of the immunosuppressive drugs.

\section{Discussion}

Histoplasma capsulatum is a dimorphic fungus occurring in a yeast phase in man and animals, and in mycelial form in the soil. ${ }^{4}$ Histoplasmosis has been reported from 40 countries around the world.' It is endemic in the eastern and central United States where histoplasmin skin tests are focally positive in $95 \%$ or more of young adults. ${ }^{4}$ Infection is acquired by inhalation of spores from the soil, especially where it is enriched with bird or bat droppings. ${ }^{4}$ Most infections produce a mild, self limiting respiratory illness, or are asymptomatic. Occasionally a chronic cavitating pulmonary disease resembling tuberculosis develops.

Haematogenous spread may lead to disseminated infection, often in patients with impaired immunity, ${ }^{235-7}$ including increasing numbers of patients with AIDS. ${ }^{8}$ Symptoms are usually non-specific and include prolonged fever, malaise, and loss of weight. ${ }^{3}$ Histological examination of biopsy and necropsy specimens shows numerous Histoplasma organisms within the macrophages of the reticuloendothelial system as well as at other sites. ${ }^{9}$ It has been suggested that an underlying defect in macrophage fungicidal function leads to invasion by the parasite. ${ }^{9}$ Granulomas are characteristically absent and there is scanty lymphocytic infiltration. Our patient probably had diffuse infection, but this was not confirmed as necropsy was not carried out.

Reports from the United States mention disseminated histoplasmosis in 27 renal transplant patients, ${ }^{310-13}$ and in one patient after bone marrow transplantation. ${ }^{14}$ The case reported here is the first reported case of histoplasmosis in a renal transplant patient in Britain that we know of. Only a few British cases of disseminated disease contracted abroad have been reported. ${ }^{1516}$ It seems likely that our patient acquired the infection in Kenya a few months before his death. Travellers to Africa may also be exposed to Histoplasma duboisii, a larger variant that predominantly affects the bones and skin.'

Gastrointestinal symptoms occur in up to $20 \%$ of patients with disseminated histoplasmosis. ${ }^{17}$ The ileum or colon, or both, are predominantly affected ${ }^{9}$ and the clinical and radiological features are often mistaken for carcinoma, ulcerative colitis, or Crohn's disease. ${ }^{18} 19$ Complications include perforation, obstruction and haemorrhage. ${ }^{1820}$ On histological examination, ulcerative and polypoid lesions are seen, with sheets of yeast filled submucosal and mucosal macrophages, ${ }^{9}$ as in our patient. Occasionally the intestine seems to be the only organ affected, suggesting the possible acquisition of viable organisms from contaminated water, though this is uncertain. ${ }^{1721} 22$

Disseminated histoplasmosis can be diagnosed from cultures of tissues and blood, histological examination, and histoplasmin skin tests or serological studies, or both. ${ }^{17}$ Skin tests and serology may be helpful in immune competent subjects, but both are often negative, or the serum antibody titres are low in those with impaired immunity. ${ }^{323}$ Detection by radioimmunoassay of $\boldsymbol{H}$ capsulatum antigen in serum and urine specimens is a potentially useful new method for diagnosing disseminated histoplasmosis. ${ }^{24}$ The test does not depend on patients' immune responses.

Fungal cultures are not invariably positive, and usually require three to four weeks for definitive identification of $\boldsymbol{H}$ capsulatum. ${ }^{23}$ A more rapid diagnosis can be obtained by histological examination. Bone marrow aspirates and liver biopsy specimens are often positive. ${ }^{52325}$ Blood smears and biopsies of other tissues have also shown the yeasts. ${ }^{3523}$

Positive staining with methenamine silver and PAS differentiates Histoplasma from Leishmania (the latter is also distinguished by observing a kinetoplast and by staining with Giemsa). Lack of capsular staining with PAS and alcian blue, and their smaller size, differen tiates Histoplasma organisms from Cryptococcus. 'Ano immunoperoxidase histoplasma antibody stain has? recently been described that accurately distinguished Histoplasma organisms from morphologically similar fungi and parasites. ${ }^{26}$ The characteristic halo around Histoplasma yeasts is an artefact, probably due to retraction of macrophage cytoplasm. ${ }^{27}$

Disseminated histoplasmosis should be a differential diagnosis in immunosuppressed patients who travel abroad and who subsequently develop prolonged fever or whose health deteriorates unexpectedly. Treatment with amphotericin B may be effective, especially if it is started early and continued for two to three months, ${ }^{36923}$ whereas in untreated cases the disease is nearly always fatal. ${ }^{3}$

\section{References}

I Binford CH, Dooley JR. Diseases caused by fungi and actinomycetes. In: Binford $\mathrm{CH}$, Connor DH, eds. Pathology of tropical and extraordinary diseases. Washington DC: AFIP, 1976:578-82.

2 Wheat LJ, Slama TG, Norton JA, et al. Risk factors for disseminated or fatal histoplasmosis. Analysis of a large urban outbreak. Ann Intern Med 1982;96:159-63.

3 Kauffman CA, Israel KS, Smith JW, White AC, Schwarz J, Brooks GF. Histoplasmosis in immunosuppressed patients. Am J Med 1978;64:923-32.

4 Goodwin RA Jr, Des Prez RM. Pathogenesis and clinical spectrum of histoplasmosis. Southern Med J 1973;66:13-25. 
5 Davies SF, Khan M, Sarosi GA. Disseminated histoplasmosis in immunologically suppressed patients. Occurrence in a nonendemic area. Am J Med 1978;64:94-100.

6 Hughes WT. Hematogenous histoplasmosis in the immunocompromised child. J Pediatr 1984;105:569-75.

7 Dismukes WE, Royal SA, Tynes BS. Disseminated histoplasmosis in corticosteroid-treated patients. JAMA 1978;240:1495-8.

8 Johnson PC, Sarosi GA, Septimus EJ, Satterwhite TK. Progressive disseminated histoplasmosis in patients with the acquired immune deficiency syndrome: a report of 12 cases and a literature review. Semin Respir Infect 1986;1:1-8.

9 Goodwin RA Jr, Shapiro JL, Thurman GH, Thurman SS, Des Prez RM. Disseminated histoplasmosis: clinical and pathologic correlations. Medicine 1980;59:1-33.

10 Wheat LJ, Smith EJ, Sathapatayavongs B, et al. Histoplasmosis in renal allograft recipients. Two large urban outbreaks. Arch Intern Med 1983;143:703-7.

11 Davies SF, Sarosi GA, Peterson PK, et al. Disseminated histoplasmosis in renal transplant recipients. Am J Surg 1979;137: 686-91.

12 Cooper PH, Walker AW, Beacham BE. Cellulitis caused by Histoplasma organisms in a renal transplant recipient. Arch Dermatol 1982;118:3-4.

13 Peterson PK, Dahl MV, Howard RJ, Simmons RL, Najarian JS. Mucormycosis and cutaneous histoplasmosis in a renal transplant recipient. Arch Dermatol 1982;118:275-7.

14 Walsh TJ, Catchatourian R, Cohen H. Disseminated histoplasmosis complicating bone marrow transplantation. Am J Clin Pathol 1983;79:509-11.

15 Partridge BM, Tanser AR. Disseminated histoplasmosis and its treatment. Postgrad Med J 1966;42:568-72.

16 Aldridge MJ, Windsor CWO, Ashton F, Fawcett FJ, Whitby JL. Case of disseminated histoplasmosis. Br Med J 1970;iv:33-4.
17 Orchard JL, Luparello F, Brunskill D. Malabsorption syndrome occurring in the course of disseminated histoplasmosis. Case report and review of gastrointestinal histoplasmosis. Am J Med 1979;66:331-6.

18 Lee KR, Lin F. Gastrointestinal histoplasmosis, roentgenographic, clinical and pathological correlation. Am J Gastroenterol 1975;63:255-65.

19 Dietz MW. Ileocecal histoplasmosis. Radiology 1968;91:285-9.

20 Nudelman HL, Rakatansky H. Gastric histoplasmosis. JAMA 1966;195:44-6.

21 Lee SH, Barnes WG, Hodges GR, Dixon A. Perforated granulomatous colitis caused by Histoplasma capsulatum. Dis Colon Rectum 1985;28:171-6.

22 Ritter C. Studies of the viability of Histoplasma capsulatum in tap water. Am J Public Health 1954;44:1299-1304.

23 Sathapatayavongs B, Batteiger BE, Wheat J, Slama TG, Wass JL. Clinical and laboratory features of disseminated histoplasmosis during two large urban outbreaks. Medicine 1983;62:263-70.

24 Wheat LJ, Kohler RB, Tewari RP. Diagnosis of disseminated histoplasmosis by detection of Histoplasma capsulatum antigen in serum and urine specimens. $N$ Engl J Med 1986;314:83-8.

25 Cooperberg AA, Schwartz J. The diagnosis of disseminated histoplasmosis from marrow aspiration. Ann Intern Med 1964; 61:289-95.

26 Klatt EC, Cosgrove M, Meyer PR. Rapid diagnosis of disseminated histoplasmosis in tissues. Arch Pathol Lab Med 1986; 110:1173-5.

27 Kirk ME, Lough J, Warner HA. Histoplasma colitis: an electron microscopic study. Gastroenterology 1971;61:46-54.

Requests for reprints to: Dr Mary T Brett, Department of Histopathology, St George's Hospital Medical School, Cranmer Terrace, London SW17 0RE, England. 